\title{
INFLUENCIA DEL ÁNGULO DE ORIENTACIÓN DE ESTRIBOS Y ESPIGONES EN LOS PROCESOS DE EROSIÓN LOCAL
}

\author{
Marta Roca Collell $^{1}$, Antonio Heleno Cardoso ${ }^{2}$, Juan Pedro Martín Vide ${ }^{1}$, Allen Bateman ${ }^{1}$
}

\begin{abstract}
Resumen:
El objeto de este trabajo es estudiar la influencia del ángulo de orientación con respecto a la corriente de un estribo de puente o espigón en la evolución temporal y en el valor final de la erosión local. Con los resultados obtenidos en el laboratorio se ha definido la curva $\theta-\mathbf{K}_{\theta}$, siendo _ el ángulo de inclinación y $\mathbf{K}_{\theta}$ la relación entre las erosiones máximas para un ángulo $\theta$ cualquiera y $\theta=$ 90ำ (estribo perpendicular a la corriente). Las conclusiones extraídas confirman las hipótesis de los trabajos más recientes que afirman que el valor máximo de erosión corresponde al ángulo de $\mathbf{9 0}$. Se ha estudiado también la evolución temporal de la profundidad de la cavidad siguiendo las formulaciones de Ettema, Franzetti et al. y Whitehouse obteniéndose, en general, buenas correlaciones.

Palabras clave: erosión, espigones de río, orientación.
\end{abstract}

\section{Introducción}

En una red de infraestructura vial los puentes son obras singulares, costosas y vitales para mantener el transporte. Un buen conocimiento de los aspectos hidráulicos de estas estructuras permite realizar un diseño más seguro y barato. Una de las causas más comunes de fallo de un puente es la erosión local producida en sus pilas y estribos. Los mecanismos de erosión alrededor de las pilas han sido tratados de forma bastante extensa en la bibliografía. Esto ha permitido desarrollar métodos de estimación de la socavación máxima y de la evolución de la erosión con el tiempo bastante aceptables dentro de determinados intervalos. Sin embargo, los fenómenos involucrados en la erosión de estribos y, por semejanza, de espigones fluviales no han sido tan ampliamente tratados en la bibliografía aunque existen numerosos ejemplos de su importancia. Según Richardson et al. (1993) en un estudio de la U.S. Federal Highway Administration del año
1973, de 383 fallos observados en puentes el $25 \%$ fueron debidos a problemas en pilas, mientras que el $72 \%$ involucraban a los estribos. Según Melville (1992) de 108 fallos de puentes observados en Nueva Zelanda durante el período 1960-1984, 29 fueron ocasionados por problemas en los estribos.

Los espigones son estructuras construidas con un cierto ángulo respecto al flujo con la finalidad de desviarlo de las zonas más críticas para impedir la erosión de las márgenes o controlar los procesos de sedimentación en el canal principal. El mantenimiento de los espigones suele ser costoso debido a las socavaciones que se producen en sus extremos.

El estudio de la erosión local, tanto en pilas como en estribos o espigones, involucra fenómenos de gran complejidad y un gran número de variables. Melville (1992) propone la siguiente expresión donde se refleja la influencia de dichas variables:

\footnotetext{
1.- Departamento de Ingeniería Hidráulica, Marítima y Ambiental. UPC.

2.- DECivil, SHRHA. Instituto Superior Técnico. Universidade Técnica de Lisboa.

Artículo recibido el 31 de mayo de 1999, y aceptado para su publicación con pequeñas modificaciones el 23 de mayo de 2000.
} 


$$
h_{\text {smáx }} / L=\left(K_{y}, K_{f}, K_{D}, K, K_{s}, K, K_{g}\right)
$$

donde $\mathrm{h}_{\text {smáx }}$ es la máxima erosión local, L la longitud del estribo y $\theta$ una función que involucra a los parámetros adimensionales $\mathrm{K}_{\mathrm{i}}$, los cuales representan los efectos de las variables siguientes:

$\mathrm{K}_{\mathrm{y}}$ : calado;

$\mathrm{K}_{\mathrm{f}}^{\mathrm{y}}$ : intensidad del flujo, $\mathrm{u} / \mathrm{u}_{\mathrm{c}}$, donde $\mathrm{u}$ es la velocidad media y uc la velocidad media crítica o de principio de movimiento;

$\mathrm{K}_{\mathrm{D}}$ : tamaño del sedimento;

$\mathrm{K}_{\sigma}$ : grado de uniformidad del sedimento;

$\mathrm{K}$ : forma del obstáculo;

$\mathrm{K}_{\theta}$ : alineación del obstáculo;

$\mathrm{K}_{\mathrm{g}}$ : geometría del canal;

En la ecuación (1) se supone que la densidad relativa del material del lecho es constante, que los efectos de viscosidad del fluido son despreciables y que no se produce erosión por contracción de las líneas de flujo.

En este trabajo nos ocupamos de la influencia de la orientación del obstáculo, $\mathrm{K}_{\theta}$, en el valor de la erosión. El coeficiente $\mathrm{K}_{\theta}$ es la relación entre la profundidad de erosión para un determinado ángulo, $\mathrm{h}_{\mathrm{s} \theta}$, y la que se obtendría en el caso que el ángulo fuera $90^{\circ} \theta$ estribo o espigón perpendicular), $\mathrm{h}_{\mathrm{s} 90^{\circ}}$ :

$$
K=h_{s} / h_{s} 90^{\circ}
$$

El ángulo se mide desde el margen aguas abajo del obstáculo.

Según algunos autores la erosión crece a medida que lo hace el ángulo. Así pues, para $\theta>90^{\circ} \mathrm{el}$ coeficiente $\mathrm{K}_{\theta}$ es mayor que la unidad. Estos estudios corresponden a Ahmad (1953), Laursen (1958), Sastry (1962) y Zaghloul (1983). Sin embargo, trabajos más recientes, Kwan (1984) y Kandasamy (1985), contradicen lo observado anteriormente y aseguran que el máximo valor de la erosión se produce para el ángulo de $90^{\circ}$ (figura 1).

Kwan y Kandasamy relacionan la diferencia de resultados con el tiempo de duración de los ensayos. La erosión en el caso de $\theta>90^{\circ}$ evoluciona más rápidamente que para ángulos inferiores. Por tanto, para un tiempo de ensayo corto, el valor de erosión máxima para $\theta>90^{\circ}$ será $h_{s \theta}>h_{s 90^{\circ}}$ No obstante, si el ensayo se deja evolucionar, los valores de erosión para el caso de estribo perpendicular

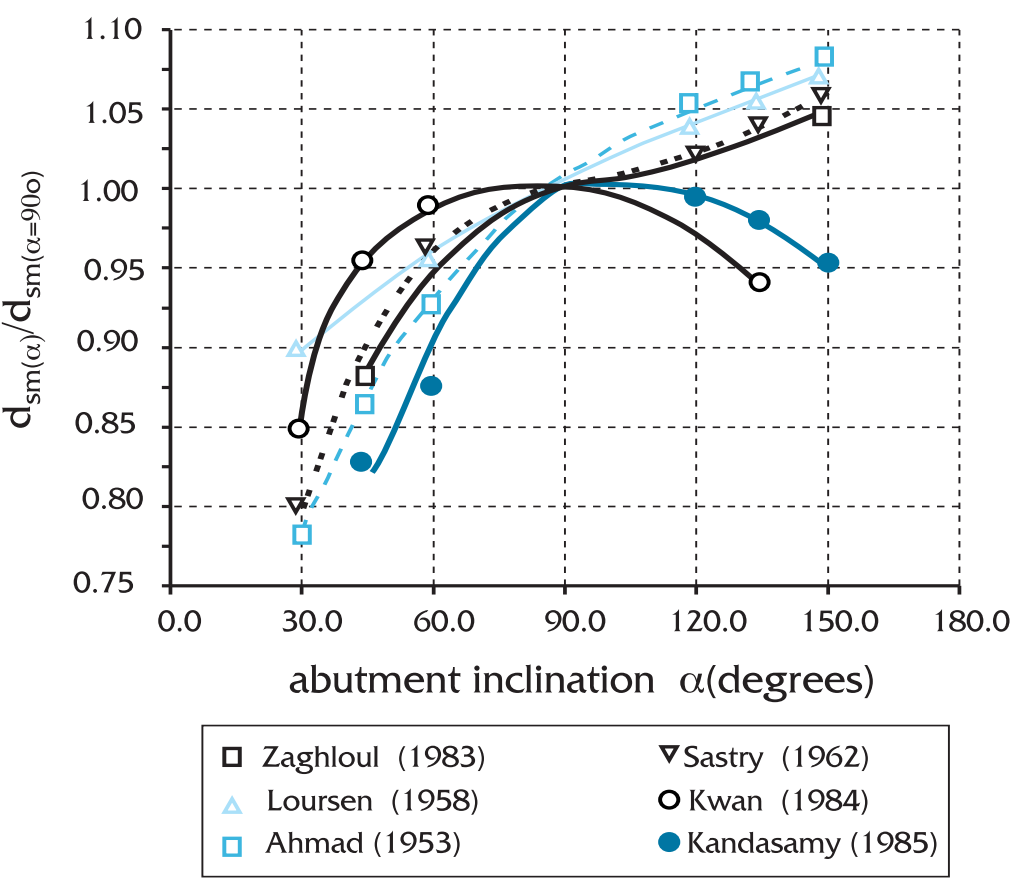

Figura 1. Efecto de la inclinación del estribo en la variación de $K_{\theta}($ Extraída de Kwan (1988))

$\left(\theta=90^{\circ}\right)$ se incrementan, con lo cual puede darse el caso que $h_{s 90^{\circ}}>h_{s \theta}$. Según este razonamiento, los ensayos anteriores a Kwan no se habrían dejado evolucionar hasta el equilibrio.

El objetivo principal de este trabajo consiste en estudiar la relación entre el factor $\mathrm{K}_{\theta} \mathrm{y}$ el ángulo de inclinación del estribo, $\theta$, a partir de datos obtenidos en ensayos de laboratorio realizados en modelo físico.

Asimismo se lleva a cabo un estudio de la evolución temporal de la erosión para los distintos ángulos ensayados siguiendo algunas formulaciones existentes, concretamente las de Ettema (1980), Franzetti et al. (1982) y Whitehouse (1997).

\section{Trabajo experimental}

El trabajo experimental se ha llevado a cabo en un canal de inclinación variable de 40,7 metros de longitud y $2 \mathrm{~m}$ de ancho situado en el pabellón de Hidráulica Marítima del LNEC (Laboratório Nacional de Engenharia Civil, Lisboa). En total se han realizado 10 ensayos con distintos ángulos de orientación: $20^{\circ}, 36^{\circ}, 54^{\circ}, 71^{\circ}, 90^{\circ}$ y sus suplementarios. En todos los casos se ha utilizado un estribo delgado de pared vertical. En cada ensayo se ha mantenido constante la proyección del estribo sobre la sección transversal, L, variando su longitud, 1, para conseguir los distintos ángulos tal como se muestra en la figura 2 . 


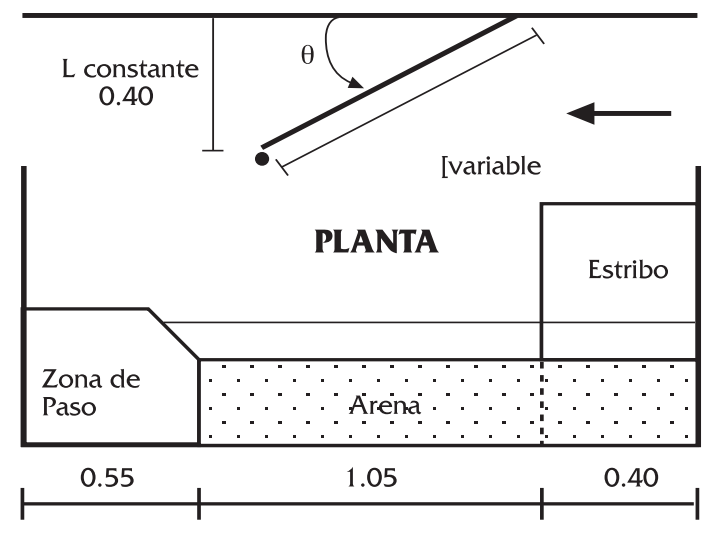

SECCIÓN TRANSVERSAL

Cotas en metros

- Puntos de medida

Figura 2. Planta y sección transversal del canal

Las condiciones bajo las cuales se han llevado a cabo los ensayos son las consideradas más desfavorables, es decir, que producen el máximo valor de erosión. Éstas son:

- ensayo de aguas claras: no hay aportes de sedimentos que puedan 'rellenar' la cavidad de erosión;

- flujo en el umbral del movimiento: $\mathrm{u} / \mathrm{u}_{\mathrm{c}}=1$, donde $\mathrm{u}$ es la velocidad del flujo y uc la velocidad crítica;

- ensayos de larga duración para aproximarse asintóticamente al valor de la erosión máxima. En nuestro caso, la duración de cada ensayo ha sido de aproximadamente una semana;

El lecho del canal está compuesto por arena con la granulometría descrita en la Tabla 1. La capa de arena tiene un espesor de $50 \mathrm{~cm}$ aproximadamente, suficiente para permitir el desarrollo total de las cavidades de erosión.

Tabla 1. Granulometría del sedimento

\begin{tabular}{|c|c|}
\hline Diámetro & $\mathbf{m m}$ \\
\hline D15,9 & 0.35 \\
D50 & 0.63 \\
D84,1 & 1.13 \\
D90 & 1.44 \\
\hline
\end{tabular}

La condición de flujo en el umbral del movimiento se traduce, para la granulometría descrita, en los siguientes valores de los parámetros del ensayo:

Tabla 2. Condiciones del ensayo

\begin{tabular}{|c|c|}
\hline Parámetro & Valor \\
\hline $\mathrm{Q}$ & $0.029 \mathrm{~m}^{3} / \mathrm{s}$ \\
$\mathrm{y}$ & $0.07 \mathrm{~m}$ \\
$\mathrm{i}$ & $0.05 \%$ \\
\hline
\end{tabular}

donde Q es el caudal circulante, y, el calado e i la pendiente del fondo de arena del canal.

Los parámetros han sido los mismos en todos los ensayos con las mínimas variaciones introducidas por el manejo de los equipos.

Para la medida de la erosión se sitúa un limnímetro en un punto muy cercano al extremo del estribo, lugar donde según experiencias precedentes se produce el máximo valor (ver figura 2) y se van realizando las distintas medidas a lo largo del tiempo. El limnímetro utilizado está pintado de color rojo en su extremo. En cada medida es introducido en el fondo de la cavidad de arena hasta que la zona de color ya no puede ser vista. De esta forma se asegura una precisión de aproximadamente $1 \mathrm{~mm}$.

\section{Resultados y discusión}

Las características de los ensayos así como los valores obtenidos se reflejan en la Tabla 3 donde $\theta$ es el ángulo del estribo, $t_{t}$ la duración total del ensayo, te el tiempo de equilibrio tal y como se define en el siguiente apartado y $\mathrm{h}_{\text {smáx }}$ el valor máximo de erosión.

Tabla 3. Valores experimentales

\begin{tabular}{|c|c|c|c|c|}
\hline Ensayo & $\theta\left(^{\circ}\right)$ & $\mathbf{t}_{\mathbf{t}}$ (horas) & $\mathbf{t}_{\mathbf{e}}$ (horas) & $\mathbf{h}_{\text {smáx }}(\mathbf{m})$ \\
\hline 1A & 19 & 167.20 & - & 0.100 \\
2A & 36 & 102.27 & 9.67 & 0.145 \\
3A & 52 & 96.75 & 36.67 & 0.244 \\
4A & 71 & 56.68 & - & 0.229 \\
5A & 90 & 141.77 & 11.50 & 0.248 \\
5B & 90 & 141.68 & 11.67 & 0.224 \\
4B & 111 & 56.73 & - & 0.212 \\
3B & 128 & 96.73 & 32.17 & 0.208 \\
2B & 143 & 102.28 & 65.17 & 0.208 \\
1B & 161 & 167.15 & - & 0.230 \\
\hline
\end{tabular}

\section{Análisis del desarrollo de la erosión}

Está aceptado de forma general que el desarrollo de la erosión a lo largo del tiempo puede dividirse en tres fases:

- inicial: empieza la erosión por aceleración del flujo al paso del estribo;

- principal: la cavidad de erosión se va desarrollando por acción del flujo descendente y del vórtice primario;

- de equilibrio: el aumento de la profundidad de erosión se convierte en insignificante; 
Estas tres fases quedan reflejadas en un gráfico $\log (\mathrm{t})-\mathrm{h}_{\mathrm{s}}$ como rectas de distintas pendientes, siendo la inclinación de la fase de equilibrio cero o muy cerca de este valor. Como ejemplo se presenta la figura 3 correspondiente al ensayo $2 \mathrm{~A}$, de ángulo $\theta=36^{\circ}$.
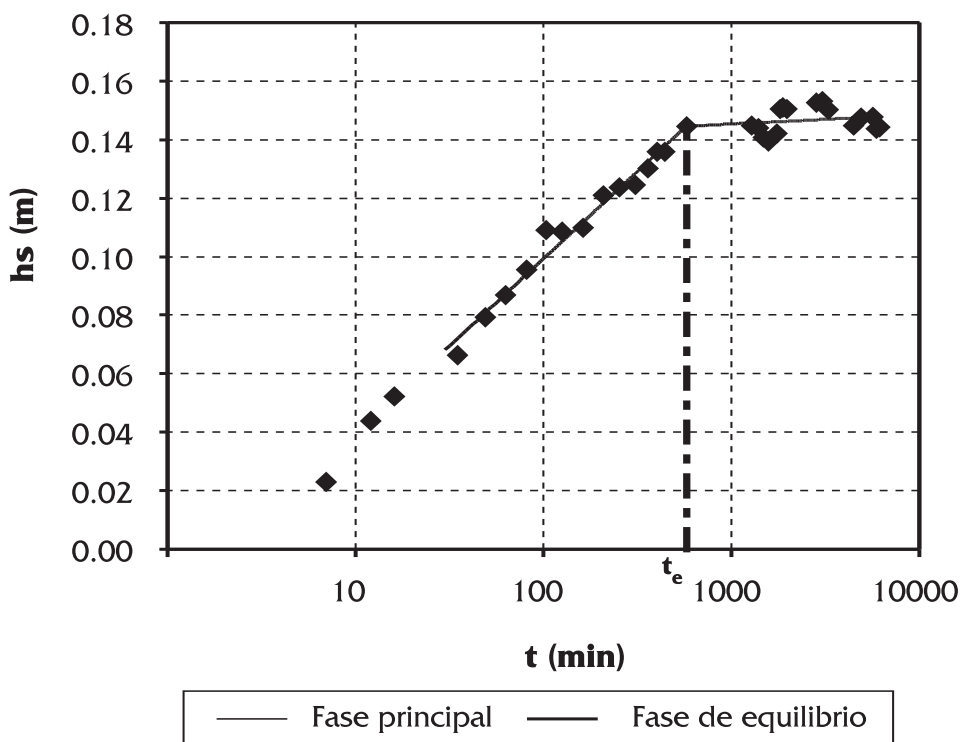

Figura 3. Relación $\log (\mathrm{t})-\mathrm{h}_{\mathrm{s}}$ para el ensayo $2 \mathrm{~A} \operatorname{con} \theta=36^{\circ}$.

La fase inicial de los ensayos resulta, en general, bastante difícil de determinar de forma ajustada. En los 10 ensayos realizados se ha considerado que los valores de la primera media hora u hora, dependiendo del caso, formaban parte de esta fase inicial.

La fase principal puede describirse mediante una recta de pendiente fijada por las medidas realizadas en los ensayos. El final queda condicionado por la fase de equilibrio que puede determinarse de manera mucho más clara que la de inicio. El punto de intersección entre estas dos rectas recibe el nombre de tiempo de equilibrio y corresponde al instante a partir del cual el ensayo se estabiliza y por tanto el valor de la erosión puede considerarse como el máximo. Un análisis detallado de los datos permite detectar un desarrollo diferenciado de la erosión durante la fase inicial. Para ángulos mayores a $90^{\circ}$ esta fase adquiere gran importancia ya que en ella se desarrolla de forma muy intensa la cavidad. Según los datos obtenidos en los ensayos los valores de erosión para 30 y 60 minutos (tiempo definido anteriormente como fase inicial) son siempre mayores para los ángulos de más de $90^{\circ}$ (excepto en los ensayos 1A y 1B). Cuando se inicia la fase principal la cavidad es pues mas profunda que para los ángulos menores a $90^{\circ}$.
La intensa erosión producida en los instantes iniciales debe explicarse a partir de la influencia de la orientación del obstáculo en el desarrollo del flujo. Aunque no ha sido objeto de estudio detallado en estos ensayos, se ha observado que la distribución del flujo originada por la perturbación que causa el estribo es significativamente distinta para los ángulos mayores y menores de $90^{\circ}$.

Para ángulos inferiores a $90^{\circ}$ las líneas de flujo sufren una 'suave' contracción creándose una estela de vórtices aguas abajo del extremo del estribo. En la superficie de la pared vertical situada aguas arriba existe también un flujo descendente que provoca erosión (figura 4.A). Para ángulos mayores de $90^{\circ}$ las líneas de flujo topan con la masa de agua 'almacenada' entre el estribo y la margen creándose una zona de gran vorticidad aguas arriba del obstáculo. Igualmente se forma una estela de vórtices desde el extremo de la pared hacia aguas abajo (figura 4.B).

Durante la fase inicial de establecimiento de las estructuras del flujo descritas de forma simplificada, las líneas de corriente en el caso B se ven más 'bruscamente' aceleradas y por tanto provocan una mayor erosión.
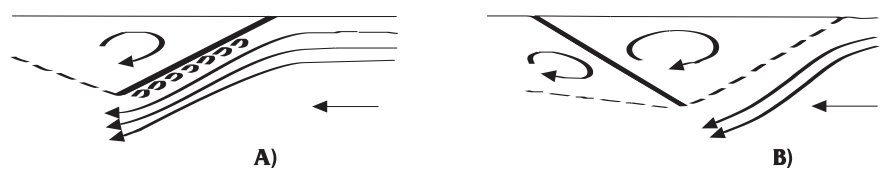

Figura 4. Esquema del flujo en estribos tipo A y B

En la fase principal las pendientes de las rectas de los ensayos tipo $\mathrm{B}\left(\theta>90^{\circ}\right)$ son menores que las de los ensayos tipo $\mathrm{A}\left(\theta<90^{\circ}\right)$. Es decir, para este último caso la erosión evoluciona más rápidamente durante la fase principal.

\section{Análisis de la curva $\theta-K_{\theta}$}

El parámetro $\mathrm{K}_{\theta}$ es el factor de relación entre $\mathrm{h}_{\mathrm{se}} \mathrm{y}_{\mathrm{s} 90^{\circ}}$. Los valores de erosión que van a utilizarse para dibujar el gráfico $\theta-\mathrm{K}_{\theta}$ corresponden al máximo medido durante el ensayo. El lugar de máxima profundidad puede coincidir con el punto donde se realizaron todas las medidas a lo largo del tiempo o bien estar ligeramente desplazado. Su localización no es tan importante como el hecho que se trate de la mayor profundidad dentro de la cavidad. El valor de la erosión correspondiente a los $90^{\circ}$ se ha obtenido como la media de los valores de los ensayos 5A y $5 \mathrm{~B}$. 
En la figura 5 puede observarse que se confirman los datos obtenidos por Kwan y por Kandasamy en los cuales el parámetro $\mathrm{K}_{\theta}$ es menor que 1 para ángulos mayores de $90^{\circ}$. Así pues, para ensayos de larga duración el valor máximo de erosión corresponde al ángulo de $90^{\circ}$.

El hecho que en el ensayo $3 \mathrm{~A}\left(\theta=52^{\circ}\right)$ el coeficiente sea superior a 1 debería interpretarse como una desviación de la medida experimental.

Los parámetros $\mathrm{K}_{\theta}$ para los ángulos mayores de $90^{\circ}$ son bastante parecidos $(0.90,0.88,0.88)$ excepto para el ángulo mayor, de $161^{\circ}(0.97)$. Si nos olvidamos de este último punto, los restantes se encuentran bastante por debajo de los medidos por Kwan y por Kandasamy. Las diferencias entre los datos obtenidos y los de estos autores pueden explicarse por el hecho de utilizar distintos tipos de estribos en los ensayos. Cada forma de estribo genera estructuras del flujo diferentes y por tanto erosiones también distintas que no son tenidas en cuenta totalmente por el factor de forma.

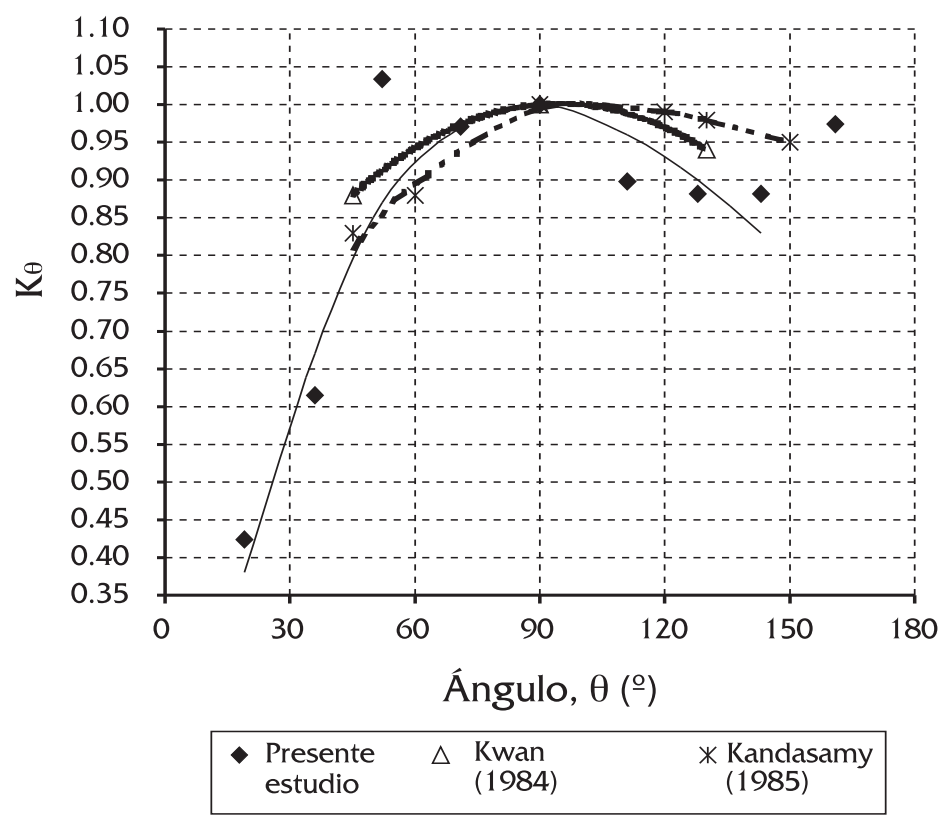

Figura 5. Variación del parámetro $K_{\theta}$ en función del ángulo

El valor de $\mathrm{K}_{\theta}$ para el ángulo de $161^{\circ}$ no es explicable a partir de las tendencias de los demás datos.

\section{Análisis de la evolución temporal de la erosión}

La evolución temporal de la erosión se estudia, como ya se ha comentado en la introducción, siguiendo las formulaciones de Ettema (1980), Franzetti et al. (1982) y Whitehouse (1997).
Ettema describe la evolución temporal de la erosión a través de una fórmula logarítmica:

$$
h_{s} / L=K_{1} \log X+K_{2}
$$

siendo $\mathrm{h}_{\mathrm{s}}$ la erosión en un instante $\mathrm{t}, \mathrm{K}_{1}$ y $\mathrm{K}_{2}$ constantes y $\mathrm{X}$ un parámetro adimensional, $\left(\mathrm{D}_{50} \mathrm{v} \mathrm{t}\right) /$ $\mathrm{L}^{3}$, donde $v$ es la viscosidad del fluido.

Franzetti et al. (1982) sugiere una función exponencial del tipo:

$$
h_{s} / h_{\text {smáx }}=1-\exp a_{1}(u t / L)^{a_{2}}
$$

donde $\mathrm{a}_{1}$ y $\mathrm{a}_{2}$ vuelven a ser constantes.

Finalmente Whitehouse propone la siguiente ecuación:

$$
h_{s} / h_{\text {smáx }}=1-\exp \left[-K(t / T)^{p}\right]
$$

donde $\mathrm{T}$ es una escala de tiempo, concretamente el tiempo en el que $h_{s}=0.632 h_{\text {smáx }}$. El valor habitual de la constante $\mathrm{K}$ es 1 .

Las medidas experimentales analizadas corresponden a la fase principal de la erosión. Los datos de las fases iniciales y de equilibrio no son utilizados. También son obviados aquellos datos cuyo valor es mayor que el $98 \%$ de la máxima erosión aún encontrándose en la fase principal. Esto se justifica por el hecho que diferencias comprendidas en ese pequeño rango se amplifican luego en los ejes logarítmicos donde se representan los datos, produciendo una distorsión en el ajuste que no se corresponde con la realidad de las medidas.

Mediante un estudio de regresión se obtienen los valores de las constantes de las distintas aproximaciones utilizadas con coeficientes de correlación superiores a $0.97,0.96$ y 0.96 para las respectivas ecuaciones. Tan sólo en un ensayo, el 1B, se presentan valores ligeramente inferiores.

No se aprecia ninguna dependencia clara entre los valores de los coeficientes de las ecuaciones 3-5 y el ángulo de inclinación.

\section{Conclusiones}

Se han realizado 10 ensayos de larga duración, de más de tres días, con 9 orientaciones distintas del estribo con la finalidad de estudiar los valores de la erosión local. Los resultados obtenidos confirman los de Kwan (1984) y Kandasamy 
(1985) en los cuales el parámetro K_ es menor que 1 para ángulos mayores de $90^{\circ}$. Así pues, para ensayos de larga duración el valor máximo de erosión corresponde al ángulo de $90^{\circ}$.

Los valores experimentales apuntan hacia la idea que los estribos con ángulos de más de $90^{\circ}$ tienen mayor erosión en la fase inicial y desarrollo más lento de ésta en la fase principal, al contrario de lo que sucede para ángulos menores a $90^{\circ}$.

Existe una buena correlación entre los datos experimentales y las ecuaciones que describen la evolución de la erosión en el tiempo. Los coeficientes de estas ecuaciones son prácticamente independientes del ángulo de inclinación.

\section{Agradecimientos}

El estudio fue realizado con el apoyo del Ministerio de Educación y Ciencia, el proyecto HID96-0971, la Xarxa d'Hidrologia Mediterrània de la Generalitat de Catalunya y en el ámbito de los proyectos PBIC/C/CEG/2355/95 y PRAXIS/ 3/3.2/CEG/32/94 financiados por la 'Fundaçao para a Ciência e Tecnologia' de Portugal.

\section{Lista de símbolos}

$\mathbf{a}_{1}$ parámetro de la ecuación de Franzetti et al.;

$a_{2}$ parámetro de la ecuación de Franzetti et al.;

D diámetro de la pila;

D diámetro de tamiz que deja pasar un porcentaje i de las partículas de sedimento;

D50 diámetro medio de las partículas de sedimento;

g gravedad;

$\mathbf{h}_{\mathrm{s}} \quad$ valor de la erosión en un determinado instante $\mathrm{t}(\mathrm{m})$;

$\mathbf{h}_{\text {smáx }}$ valor máximo de la erosión en la fase de equilibrio (m);

$\mathbf{h}_{\mathrm{s} \theta} \quad$ valor de la erosión para un determinado ángulo ( (m);

$\mathbf{h}_{\mathrm{s90}} \quad$ valor de la erosión para el ángulo de $90^{\circ}(\mathrm{m})$;

i inclinación del canal donde se realizan los ensayos;

$\mathbf{K}_{1} \quad$ parámetro de la ecuación de Ettema;

$\mathbf{K}_{2}$ parámetro de la ecuación de Ettema;

$\mathbf{K}_{\mathbf{D}}$ parámetro adimensional que representa los efectos del tamaño del sedimento;

$\mathbf{K}_{\mathrm{f}}$ parámetro adimensional que representa los efectos de la intensidad del flujo, $\mathrm{u} / \mathrm{u}_{\mathrm{c}}$, donde $\mathrm{u}$ es la velocidad media y $\mathrm{u}_{\mathrm{c}}$ la velocidad media crítica;

$\mathbf{K}_{\mathbf{i}} \quad$ parámetro adimensional que representa los efectos de la variable i;

$\mathbf{K}_{\mathrm{g}} \quad$ parámetro adimensional que representa los efectos de la geometría del canal;

$\mathbf{K}_{\mathrm{L}}$ parámetro adimensional que representa los efectos de la longitud del estribo L, o diámetro de la pila $\mathrm{D}$;

$\mathbf{K}_{\mathrm{s}} \quad$ parámetro adimensional que representa los efectos de la forma del obstáculo;

$\mathbf{K}_{\mathbf{y}}$ parámetro adimensional que representa los efectos del calado;

$\mathbf{K}_{\theta} \quad$ parámetro adimensional que representa los efectos del ángulo de inclinación del estribo;

$\mathbf{K}_{\sigma} \quad$ parámetro adimensional que representa los efectos del grado de uniformidad del sedimento;

K parámetro de la ecuación de Whitehouse;

L proyección del estribo en la dirección perpendicular al flujo $(\mathrm{m})$;

l longitud del estribo (m);

p parámetro de la ecuación de Whitehouse;

t tiempo;

$\mathbf{t}_{\mathrm{e}} \quad$ tiempo de equilibrio;

$\mathbf{t}_{\mathbf{t}} \quad$ tiempo total de duración del ensayo;

u velocidad media de aproximación del flujo $(\mathrm{m} / \mathrm{s})$;

$\mathbf{u}_{\mathbf{c}} \quad$ velocidad crítica de las partículas $(\mathrm{m} / \mathrm{s})$;

X parámetro adimensional de la ecuación de Ettema;

$\mathbf{y}$ calado (m);
$\boldsymbol{\theta}$ ángulo de inclinación del estribo medido en grados desde el margen de aguas abajo;

$v \quad$ viscosidad del fluido;

$\varphi \quad$ función;

\section{Referencias}

Ahmad, M. (1953) Experiments on designs and behaviour of spur dikes. Proc. IAHR. Conf. Minnesota, pp 145-159.

Cardoso, A.H. Eros(es localizadas junto de espor(es fluviais e de encontros e pilares de pontos. Informe del CEHIDRO, Instituto Superior Técnico, UTL, Lisboa (versión provisional).

Cardoso, A.H., Bettes, R. Time evolution and effect of channel geometry on local scour at bridge abutment. Journal of Hydraulic Engineering (aceptado para su publicación).

Dongol, D.M.S. (1984) Local scour at bridge abutments. Tesi de doctorado. University of Auckland. Department of civil engineering. Report $n^{\circ} 544$.

Ettema, R. (1980). Scour at bridge piers. University of Auckland. Department of civil engineering. Report $\mathrm{n}^{\circ} 216$.

Franzetti, S., larcan, E., Mignosa, P. (1982). Influence of tests duration on the evaluation of ultimate scour around circular piers. International Conference on the Hydraulic Modelling of Civil Engineering Structures, paper G2, 
Coventry, England.

Kandasamy, J.K. (1985). Local scour at skewed abutments. University of Auckland. Department of civil engineering. Report $\mathrm{n}^{\mathrm{0}} 375$.

Kandasamy, J.K. (1989) Abutment scour. Tesi de doctorado. University of Auckland. Department of civil engineering. Report $\mathrm{n}^{\circ} 458$.

Kothary, U.C., Ranga Raju, K.G., Garde, R.J. (1992) Livebed scour around cylindrical bridge piers. Journal of Hydraulic Research. Vol. 30, nº 5. pp 701-715.

Kwan, T.F. (1984) Study of abutment scour. University of Auckland. Department of civil engineering. Report $\mathrm{n}^{\circ}$ 328.

Kwan, T.F. (1988) A study of abutment scour. Tesi de doctorado. University of Auckland. Department of civil engineering. Report $\mathrm{n}^{\circ} 451$.

Laursen, E.M. (1958) Scour at bridge crossings. Iowa. Highway Research Board Bulletin, $n^{\circ} 8$, Iowa Institute of Hydraulic Research, State University of Iowa, USA.

LNEC (1982) Canal de inclinaç(o variável. Descriçco do tanque de montante, canal propiamente dito, tanque de jusante e circuito de retorno. Ministerio de Habitaç(o e obras públicas. Laboratorio Nacional de Engenharia Civil.

Melville, B.W. (1992). Local scour at bridge abutments. Journal of Hydraulic Engineering, ASCE. Vol. 118, $\mathrm{n}^{\circ}$ 4 April, pp 615-631.

Melville, B.W. (1995). Bridge abutment scour in compound channels. Journal of Hydraulic Engineering, ASCE. Vol. 121, n 12 December, pp 863-868.

Melville, B.W. (1997). Pier and abutment scour: integrated approach. Journal of Hydraulic Engineering, ASCE. Vol. 123, n 2, February, pp 125-136.

Richardson, E.V., Harrison, L.J., Richardson, J.R. Davies, S.R. (1993). Evaluating scour at bridges. Federal Highway Administration, Pub. FHWA-P-90-017, Virginia, USA.

Whitehouse, R.J.S. (1997). Scour at marine structures: a manual for engineers and scientists. HR Wallingford Research, report SR417, Wallingford, UK. 


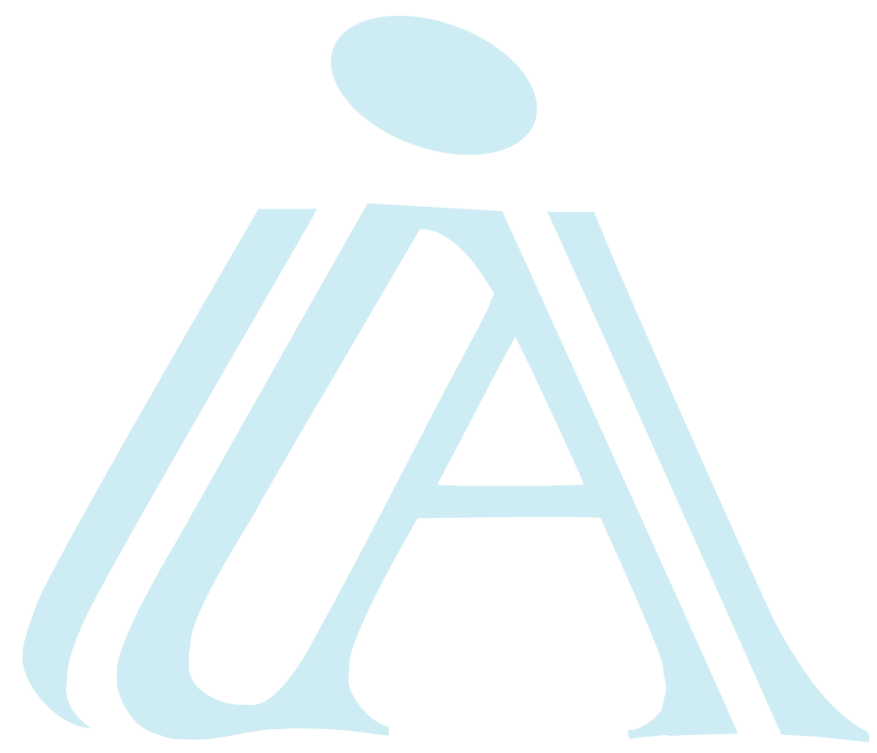

